\title{
The Real Time Processor LDV Signals with an Acoustooptic Light Deflector and a Position Sensitive Device*
}

\author{
Noboru Nakatani*, Atsunori Uehara* and Tomoharu Yamada*
}

This paper describes the signal processor by the use of an acoustooptic light deflector for processing of LDV signals in real time and with high speed. The position sensitive device using the lateral photo-effect of a semiconductor is investigated for real time measurement of the displacement of a beam spot with large intensity variation. The frequency response of the processor is $100 \mathrm{kHz}$ and the frequency resolution, $5 \mathrm{kHz}$. As the processor is open loop system, it can process LDV signals in wide frequency range and with high speed compared with a conventional frequency tracker. Using the flow around a circular cylinder, it is demonstrated that this signal processor can be used for continuous and discontinuous LDV signals and is a powerful tool for highly instationary flow.

Key Words: signal processing, optical computing, laser Doppler velocimeter

\section{Introduction}

When there are so many particles in the fluid that LDV signals are obtained almost continuously and when the LDV signals are noisy, the signals have been processed by a frequency tracker. The tracker, however, has not high frequency response enough to follow large and fast variation of the LDV signals because it has closed loop circuit. When there are so few particles in the fluid that LDV signal is discontinuous, the tracker is out of

† IECON'84 で発表 (昭 $59 \cdot 10$ )

* Faculty of Engineering, Osaka University, Suita 大阪大学工学部 吹田市山田丘 2-1

(Received November 21, 1984)

(Revised May 25, 1985) capture range for dropout signal. In this study we make possible to process continuous or discontinuous LDV signals in real time and with high speed by the use of an acoustooptic light deflector (AOD).

Acoustooptical interaction has been recently used to perform real time optical Fourier analysis of wide band electrical signals. Various types of spectrum analyzers have been realized ${ }^{1)-3)}$. As the linear photodetector arrays have been used to detect the position of a laser beam spot in these analyzers, the time required for real time processing has been limited by scanning time of the array. To overcome the limitations, the position sensitive device (PSD) using the lateral photoeffect of a semiconductor is investigated for real time measurement of the displacement of the laser beam spot.

Using the flow around a circular cylinder, the characteristics of this LDV signal processor is investigated.

\section{Real Time Signal Processor}

The configuration of an acoustooptical signal processor is shown in Figure 1. The LDV used is shown in Figure 2. The LDV is differential type. The optical frequency of one of two beams incident to flow field is shifted by $40 \mathrm{MHz}$ with the acoustooptic modulator (AOM) to satisfy Bragg diffraction condition of the AOD shown in Figure 1. The output signal frequency from the photomultiplier becomes $\left(40 \mathrm{MHz}+f_{D}\right)$ by Doppler frequency shifting $f_{D}$ in fluid. The frequency $f_{D}$ is given as

$$
f_{D}=(2 n v \sin \theta) / \lambda
$$


Fig. 1 Schematic diagram of the acoustooptic processor, Lens $l_{1}$ : focal length $F_{3}=1 \mathrm{~m}$, laser: $\mathrm{He}-\mathrm{Ne}$ laser, power $5 \mathrm{~mW}$, beam diameter 0.9 $\mathrm{mm}$ (the width of $1 / e^{2}$ intensity)
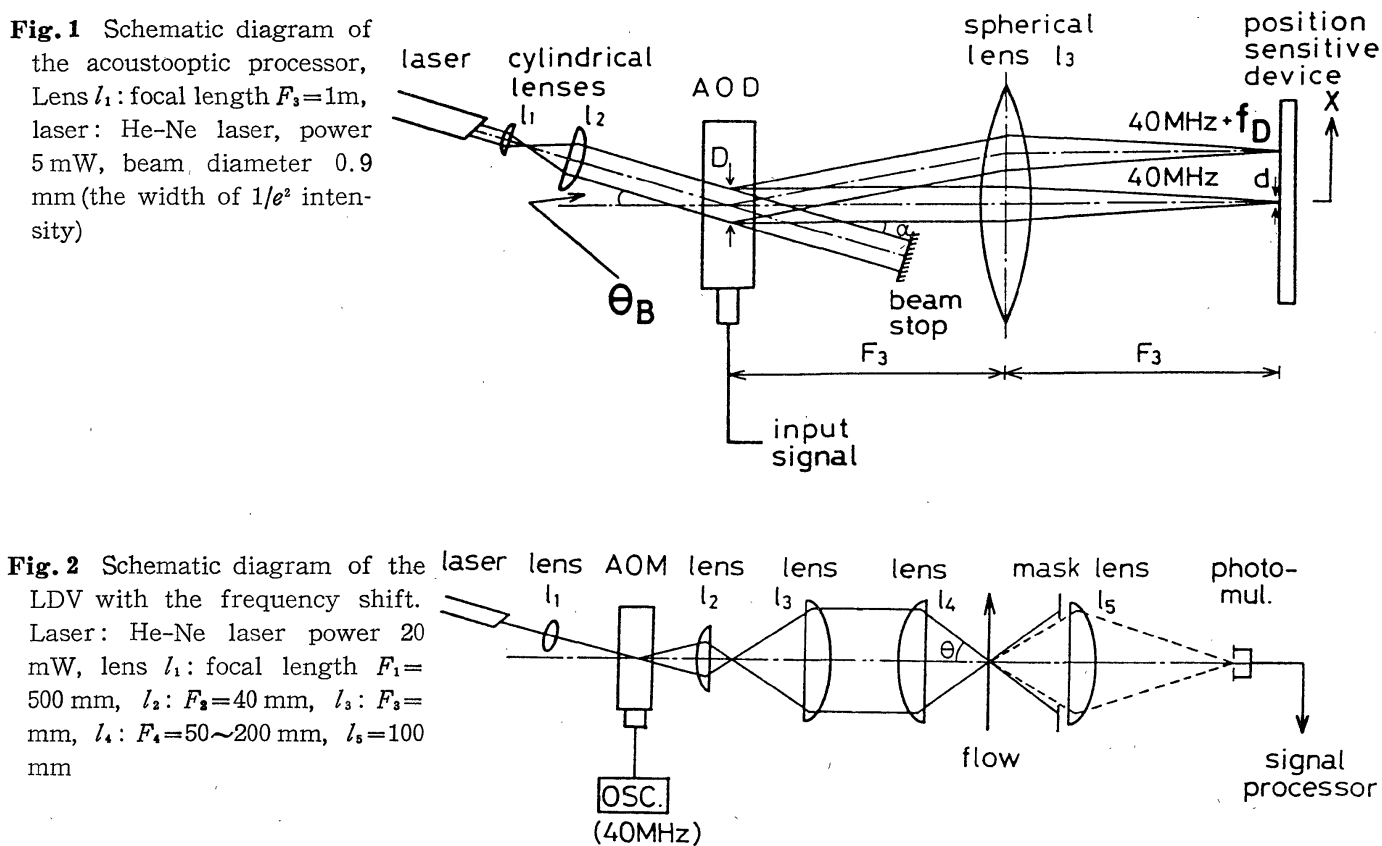

where $v$ : fluid velocity, $\lambda$ : light wavelength, $n$ : refractive index of fluid. The output signal is put in the AOD through a power amplifier. The signal is converted by the transducer into an acoustic wave which propagates along the AOD with velocity $v_{s}$. The phase grating is produced by the acoustic beam. A collimated laser beam crosses the AOD and is deflected by the acoustic beam. The deflection angle of laser beam is proportional to the frequency $\left(40 \mathrm{MHz}+f_{D}\right)$. The deflection beam passes through Fourier-transform-lens $l_{3}$ and the beam deflection is transformed to the displacement $x$ given as

$$
x=\lambda\left(F_{3} / v_{s}\right) f_{D}
$$

When the light source is He-Ne laser $(\lambda=632.8$ $\mu \mathrm{m})$ and the focal length $F_{3}$ of the lens $l_{3}$ is $1 \mathrm{~m}$, the displacement $x$ for AOD $1\left(\mathrm{TeO}_{2}\right.$, off $\langle 110\rangle$ type, $\left.v_{s}=6.5 \times 10^{2} \mathrm{~m} / \mathrm{s}\right)$ is given as

$$
x \mu \mathrm{m}=9.74 \times 10^{-1} f_{D}(\mathrm{kHz})
$$

for AOD $2\left(\mathrm{SF} 4\right.$ glass, $\left.v_{s}=3.72 \times 10^{3} \mathrm{~m} / \mathrm{s}\right)$,

$$
x \mu \mathrm{m}=1.70 \times 10^{-1} f_{D}(\mathrm{kHz})
$$

The time $\tau$ required for the acoustic beam to pass through the laser beam of the width $D$ is called access time. It is given as

$$
\tau=D / v_{s}
$$

To obtain the real time frequency spectra of LDV signal, it is required that the acoustic wave of the LDV signal continues during the access time and that the signal frequency remains constant. When there are so many particles that the LDV signal is continuous, the measurable maximum velocity is not limited by the access time. However, when there are so few particles that the LDV signal is discontinuous, the measurable maximum velocity is limited by the access time $\tau$ and is given as

$$
v_{\max }=l_{x} / \tau
$$

where $l_{x}$ is the length of the sampling volume in the velocity direction. In both cases of the continuous and discontinous LDV signals, the allowable frequency of velocity variation is given as

$$
f_{\tau}=1 / \tau
$$

The measurable maximum flow velocity and the frequency response are shown in Table 1. Each value in this table is calculated for $l_{x}=50 \mu \mathrm{m}$. Of course, it depends on $l_{x}$ value determined by a LDV optical system. The ambiguity width of the beam position $d$ due to finite residence time of the acoustic wave is inversely proportional to the width $\mathrm{D}$ of the laser beam incident to the AOD. However, when $\mathrm{D}$ value is increased for decrease of the ambiguity width, the frequency response and the measurable maximum velocity becomes 
Table 1 Measurable maximum flow velocity $V_{\max }$ and frequency response $f_{\text {. }}$ $l_{x}=50 \mu \mathrm{m}, \mathrm{AOD} 1 \mathrm{TeO}_{\mathrm{s}}$, off $\langle 110\rangle$ type, $V_{s}=6.50 \times 10^{2} \mathrm{~m} / \mathrm{s}$, AOD $2: \mathrm{SF} 4$ glass, $V_{s}$ $=3.72 \times 10^{3} \mathrm{~m} / \mathrm{s}$

\begin{tabular}{l|lr|l|l|l|l}
\hline \hline \multicolumn{2}{r}{$\mathrm{D}(\mathrm{mm})$} & 0.9 & 0.8 & 3.6 & 7.2 \\
\hline \multirow{4}{*}{ AOD 1 } & $\tau$ & $(\mu \mathrm{s})$ & 1.38 & 2.77 & 5.54 & 11.1 \\
& $f_{\tau}$ & $(\mathrm{kHz})$ & 722 & 361 & 181 & 90.3 \\
& $V_{\max }$ & $(\mathrm{m} / \mathrm{s})$ & 36.1 & 18.1 & 9.0 & 4.5 \\
\hline \multirow{3}{*}{$\mathrm{AOD} 2$} & $\tau$ & $(\mu \mathrm{s})$ & 0.242 & 0.484 & 0.968 & 1.94 \\
& $f_{\tau}$ & $(\mathrm{MHz})$ & 4.13 & 2.07 & 1.03 & 0.517 \\
& $V_{\max }(\mathrm{m} / \mathrm{s})$ & 206.7 & 103.3 & 51.7 & 25.8 \\
\hline
\end{tabular}

small.

\section{Measurement of Displacement of}

\section{A Laser Beam Spot}

When LDV signal suffers from dropout, the diffraction beam intensity of the AOD is 0 . And when the scattering particle passes near end of LDV sampling volume, amplitude of LDV signal is small and then diffraction beam intensity of the AOD is weak. Displacement of a beam spot with large intensity variation, such as in the above described cases, could not be measured by the

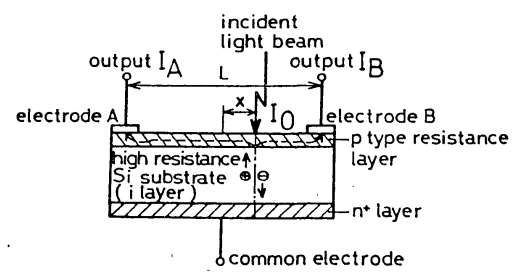

(a)

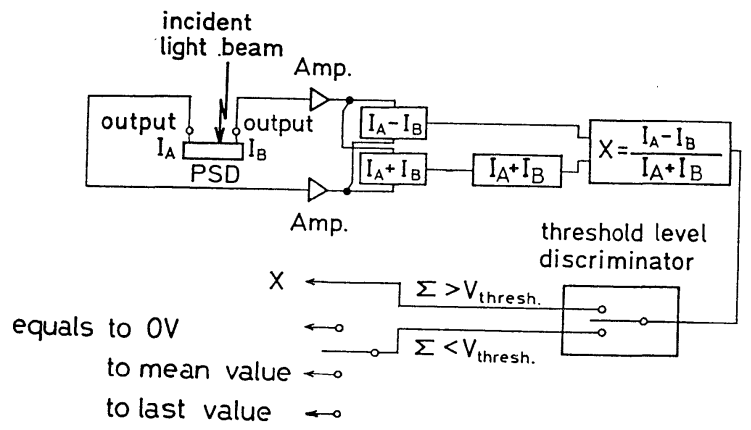

(b)

Fig. 3 Schematic diagram of a one-dimensional position sensitive device using the lateral Photo-effect of a semiconductor (PSD). (a): Part of detection, (b) : signal processing system. $\boldsymbol{\Sigma}=I_{\boldsymbol{A}}+I_{B}$ conventional PSD using the lateral photo-effect. In this study processing of the PSD output signals is investigated in order to make it possible to process discontinuous LDV signals with large variation of amplitude.

The schematic diagram of the PSD is shown in Figure 3. The output of the PSD is given as

$$
\begin{aligned}
X & =\left(I_{A}-I_{B}\right) /\left(I_{A}+I_{B}\right) \\
& =2 x / L
\end{aligned}
$$

where $I_{A}$ and $I_{B}$ : electric current in legs $A$ and $B$, $x$ : beam position, $L$ : length of p-type resistance layer. To exclude the influence of the intensity variation of the light beam on the position measurement, the difference $\left(I_{A}-I_{B}\right)$ is divided by the total current $\left(I_{A}+I_{B}\right), X$ is proportional to the position of beam spot from center. When voltage $\Sigma$ corresponding to $\left(I_{A}+I_{B}\right)$ is above $5 \mathrm{~V}$, the proportional constant is kept constant. However, when $\Sigma$ is below $5 \mathrm{~V}$, the proportional constant is not kept constant. Under the condition $\Sigma>5 \mathrm{~V}$, the relation between the PSD output $X$ and the

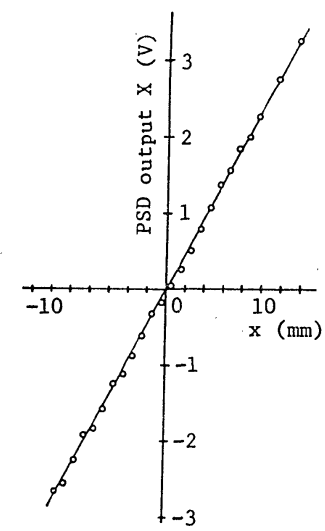

Fig. 4 The curve of output voltage $X$ of the PSD against position $x$

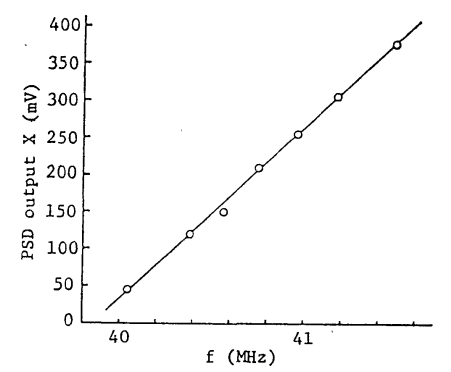

Fig. 5 The curve of output voltage $X$ of the PSD against driving frequency of the AOD 1 
N. Nakatani, A. Uehara, and T. Yamada: The Real Time Processor LDV Signals with an Acoustooptic Light Deflector

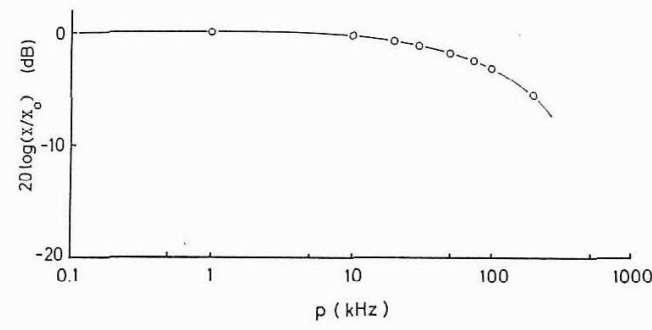

Fig. 6 Frequency response of PSD. p: Frequency of signal frequency variation, $X$ : output voltage of $P S D, X_{0}$ : maximum voltage against $p$.

AOD driving frequency obtained from equation (4) and Figure 4 is shown in Figure 5. Both curves are fit to linear curves. The position resolution of the PSD is $5 \mu \mathrm{m}$ and the frequency resolution $5 \mathrm{kHz}$. FM signals are put into the $\triangle O D 1$ to investigate the response of the PSD. The input signal is given as

$$
A \sin \{\omega t+(\Delta \omega / p) \sin p t+\varphi\}
$$

where $\omega_{c}$ is carrier frequency, $40 \mathrm{MHz}, \Delta \omega_{D}$ : variation width of shifting frequency, $70 \mathrm{kHz}, p$ : frequency of signal frequency variation, $\varphi$ : phase of signal. The response curve is shown in Figure 6 . The frequency response is $100 \mathrm{kHz}$ from the figure. When the measurable frequency range of a conventional frequency tracker is in the resolution $5 \mathrm{kHz}$, the frequency response is about $100 \mathrm{H}_{z}-1$ $\mathrm{kHz}^{4}$. Therefore this signal processor using the AOD is superior to signal processing with high speed.

When LDV signal suffers from dropout, the denomiator of $X$ in equation ( 8 ), $\Sigma$, is 0 and then $X$ output signals have noise as shown in Figure 7 (a) and (b). When LDV signal is weak, the proportional constant is not kept constant. The discriminator is used for excluding the noise and influence on variation of the proportional constant.

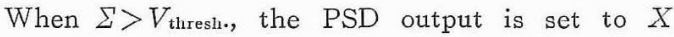
value. When $\Sigma<V_{\text {thresh }}$, the output is set to mean velocity, or to last value before signal dropout. When $V_{\text {thresh. }}$ is $5 \mathrm{~V}$ and the output is set to $0 \mathrm{~V}$, the PSD output pattern for signal with dropout is shown in Figure $7(\mathrm{c})$. The noise shown in Figure $7(b)$ is excluded. The response time of the signal processor $10 \mu \mathrm{s}$ is longer than the access time. Therefore, when LDV signal is discontinuous, the

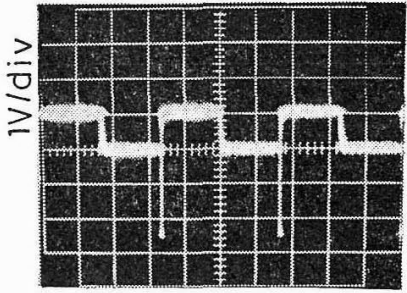

(a)

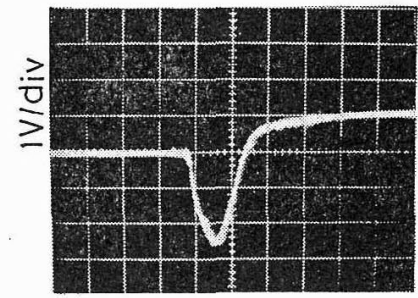

(b)

$4 \mu \mathrm{s} / \mathrm{div}$

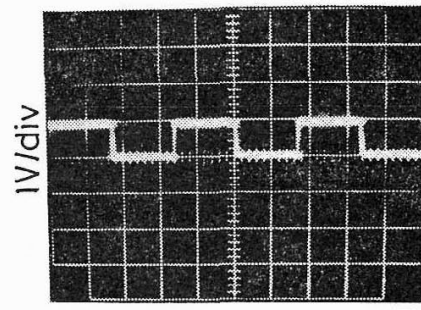

$0.2 \mathrm{~ms} / \mathrm{div}$

(c)

Fig. 7 Oscilloscope patterns of output $X$ of PSD. (a): With $\Sigma \neq 0$ and $\Sigma=0$, (b) : noise part of (a) with $\Sigma=0,\left(\right.$ c): $V_{\text {thresh. }}=5 \mathrm{~V}$

measurable veloctiy depends on the response time. The velocity becomes about $4 \mathrm{~m} / \mathrm{s}$ in the AOD 1 and $l_{x}=50 \mu \mathrm{m}$.

\section{Experimental Results of Karman Vortex Flow Measurement}

The flow below a circular cylinder in a rectangular duct is measured. The section of the duct is $5 \times 25 \mathrm{~mm}$. The diameter of the cylinderds is $1 \mathrm{~mm}$. The fluid is water. The scattering particles are $\mathrm{TiO}_{2}$. Reynolds number defined on terms of the diameter of the cylinder is 1625. In the LDV of Figure $2, F_{3}$ is $50 \mathrm{~mm}$ and $\sin \theta, 0.15$. The experimental results obtained by the use of the signal processor are shown in Figure 8 . When LDV signal is continuous, the Karman vortex frequency can be seen clearly on the oscilloscope as shown in Figure 8(a). The frequency of the velocity 


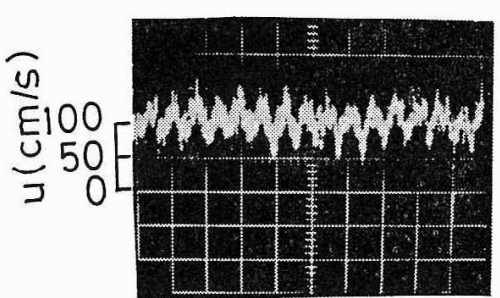

$5 \mathrm{~ms} / \mathrm{div}$

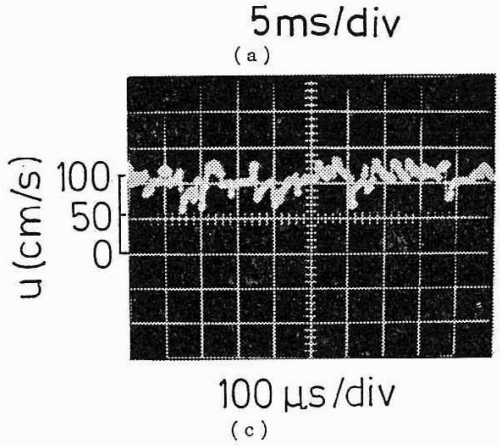

variation is $320 \mathrm{~Hz}$. The Strouhal number $S=f_{s t}$ $d_{s} / \nu$ ( $\nu$; kinematic viscosity) obtained from the frequency $f_{s t}$ becomes 0.22 . This value agrees well with that of the previous experiment ${ }^{5)}$ using a frequency tracker. In the dropout ratio 0.4 , in which a frequency tracker can not follow variation of the LDV signals, the Karman vortex flow is measured by the processor using the AOD. The expreimental results are shown in Fig. 8 (b), (c) and(d). The periodic frequency can not be observed clearly on the oscilloscope. However, when the velocity variation is analyzed by a spectrum analyzer, the spectrum peak for the frequency can be observed clearly. The spectra of Figure $8(\mathrm{c})$ and (d) are shown in Figure 9. The peak value of the spectrum in the dropout ratio 0.4 of (c) is about half of that in the dropout ratio of 0 of $(a)$. In the case of (c) the relation between the spectra with dropout $S(\omega)$ and without dropout $S_{0}(\omega)$ is given as

$$
S(\omega)=\nu S_{0}(\omega)
$$

where $\nu$ is dropout ratio. Therefore, in the case of (c) the influence of the dropout on the spectra can be excluded easily compared with those in the case of (b) and (d).

\section{Conclusions}

To process LDV signals in real time and with high speed, the signal processor is developed by
Fig. 8 Influence of signal dropout on observation of Karman vortex. $\mathrm{Re}=10000$. (a): Output without dropout (b) : output is set to zero during dropout, (c) : output is set to mean flow velocity during dropout, (d) : output is set to last value during dropout.

\section{(b)}

$\mathrm{ms} / \mathrm{div}$

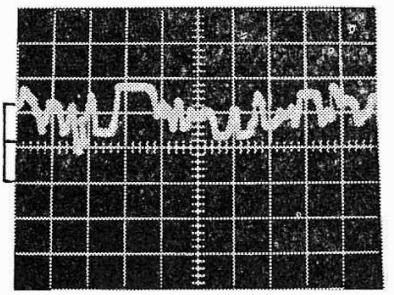

$100 \mu \mathrm{s} / \mathrm{div}$

(d)
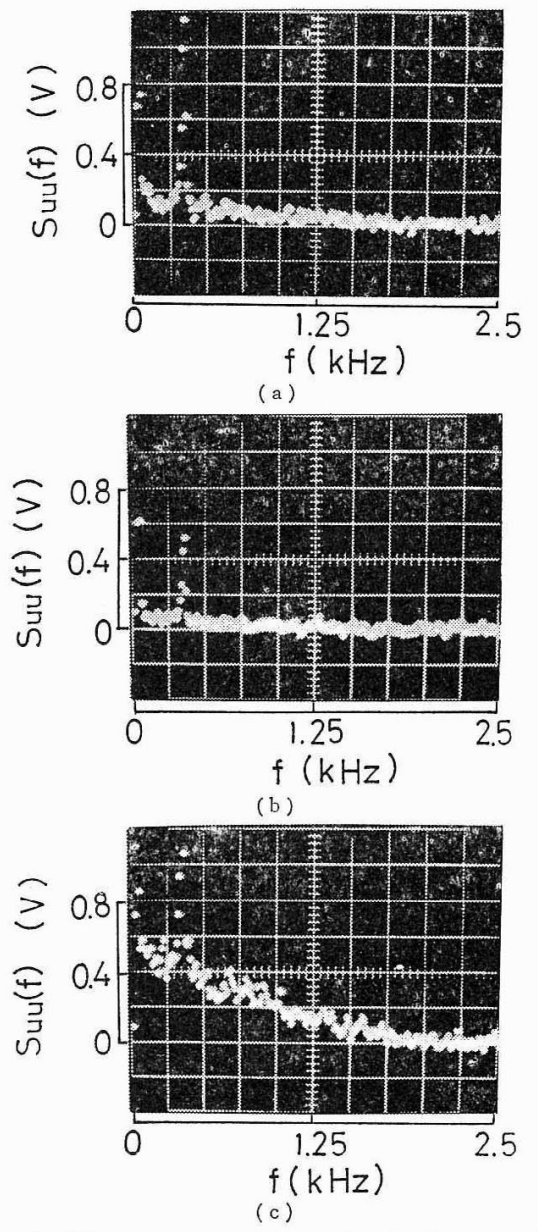

Fig. 9 The power spectra of velocity variations shown in Figure 8. (a): For (a), (b): for (c), (c) : for (d). 
N. Nakatani, A. Uehara, and T. Yamada: The Real Time Processor LDV Signals with an Acoustooptic Light Deflector

the uses of an acoustooptic light deflector (AOD) and the position sensitive device (PSD) using the lateral photo-effect of a semiconductor. The frequency response of the processor is $100 \mathrm{kHz}$ and the frequency resolution, $5 \mathrm{kHz}$. As the processor is open loop system, it can process LDV signals in wide frequency range and with high speed compared with a conventional frequency tracker. For discontinuous LDV signals the measurable maximum velocity of the processor depends on the access time of the $\mathrm{AOD}$ and the frequency response of the processor. For continuous LDV signals it is independent of them. Using the flow around a circular cylinder, it is demonstrated that the processor is a powerful tool for highly instationary flow.

\section{Acknowledgements}

The authors express their thanks to Hamamatsu Photonics Co., Ltd., for cooperation of making the position sensitive device.

This work was partially supported by the Grantin-Aid for Special Project Research from the
Ministry of Education in Japan.

\section{References}

1) A. Alippi, P. Palam, L. Palmieri, G. Socino and E. Verona: Real Time Acoustooptical Spectrum Analyzer Through Unguided Light-Surface Acoustic Waves Interaction, Optics Communications, 351, 37/41 (1980)

2) D. Mergeian, E. C. Malarkey, R.P. Pautienus, J.C. Braley, G.E. Marx, L.D. Hutcheson and A. I. Kellner: Operational Integrated Optical r. f. Spectrum Anayzer, Applied Optics, 19-8, 3033/3034 (1980)

3) J.P.Y. Lee: Acoustooptic Spectrum Analysis of Radar Signals Using an Integrating Photodetector Array, Applied Optics, 20-5-14, 595/600 (1981)

4) N. Nakatani, R. Yorisue and T. Yamada: A Laser Doppler Velocimeter with Variable Frequency Shift for ,Combustion Flow Measurement, Tech. Report of the Osaka University, 27, 1382, 463/473 (1977)

5) N. Nakatani, T. Nishikawa, Y. Yoneda and T. Yamada: The Flow Analysis of the Vortex Shedding Flowmeter by the Laser Doppler Velocimeter, Proc. of IMEKO Symp. on Flow Measurement and Control in Industry, Tokyo, Japan, 103/108 (1978)

6) P. Buchhave and W.K. Geroge: The Measurement of Turbulence with the Laser Doppler Anemometer, Annual Review of Fluid Mechanics., 11, 443/503 (1979) 